\title{
ESTUDO DA AFLATOXINA NO AMENDOIM, DA COLHEITA À INDUSTRIALIZAÇÃO, NA REGIÃO DE MATÃO, S. P.*
}

HOMERO F'ONSECA **

\section{RESUMO}

Neste trabalho foi investigada, na região de Matão, a incidência de aflatoxina no amendoim (Arachis hypogaea L.) em três estágios de seu ciclo de industrialização: a) ao ser entregue à fábrica: Épocas I e II; b) durante seu armazenamento: Epocas III e IV, e c) após a extração do óleo (farelo): Epocas $\mathrm{V}$ e VI. Em cada Estágio foram feitas duas coletas de 10 amostras cada, num total de 40 amostras de amendoim e 20 de farelo.

Dos resultados concluiu-se que: a) a maioria das amostras, representando $85 \%$ do total, continha aflatoxina; b) o nível nas amostras, em termos de aflatoxina $B_{1}$, elevou-se continuamente da Epoca I para a Epoca VI: médias de 0,06 até $1,22 \mathrm{ppm}$ com média geral de 0,44 ppm; c) as boas práticas de secagem e adequado armazenamento concorrem decisivamente para manter um nível baixo de aflatoxina, e d) o lavrador entrega amendoim já tóxico à fábrica.

\section{INTRODUÇÃO}

A descoberta da aflatoxina veio aumentar o número das substâncias carcinogênicas conhecidas. O problema das hepatotoxinas e dos hepatocarcinógenos, presentes em tortas e farelos de amendoim é bem mais antigo do que se possa imaginar e antecede bastante às perdas de peruzinhos (BLOUNT, 1961), marrequinhos e pintinhos (ASPLIN e CARNAGHAN, 1961), porcos (LOOSMORE e HARDING, 1961) e bezerros (LOOSMORE e MARKSON, 1961) relatados de 1960 para cá. PAGET, citado por SCHOENTAL (1967), descreveu em 1954 um surto de doenças hepáticas em cobaias, em vários laboratórios ingleses e os atribuiu às rações utilizadas. Outros perdem-se no passado por não terem tido explicações plausíveis na época.

* Entregue para publicação em 10/12/1976

* Departamento de Tecnologia Rural. 
Quando STEVENS et alii (1960) descreveram o aparecimento de uma nova doença em peruzinhos, começou a ser escrita a história da aflatoxina. As aves morriam geralmente dentro de uma semana sendo a perda de apetite, diminuição da mobilidade, fraqueza das asas e das pernas, seus principais sintomas. A necrópsia revelava sempre lesões necróticas no fígado, congestionamento dos rins, bem como proliferação dos ductos biliares. Não sendo possível isolar nenhum patógeno, suspeitou-se que ela deveria ser de origem alimentar visto que, com a mudança da ração, freqüentemente cessava a mortalidade, fato esse comprovado por SMITH (1969). A doença foi simultaneamente descrita também por SWARBRICK (1960) e BLOUNT (1960) entre outros, tendo sido batizada por BLOUNT (1961) de "Doença "X" dos perus" e foi considerada responsável pela morte de mais de 100.000 peruzinhos, ocorridos entre maio e agosto de 1960. SARGEANT et alii (1961) relataram que a causa era uma toxina produzida pelo fungo Aspergillus flavus Link, tendo essa substância recebido o nome de aflatoxina. A princípio foram identificadas quatro substâncias tóxicas (HARTLEY et alii, 1963) que expostas à luz ultravioleta emitiam fluorescências azuis e esverdeadas e foram denominadas de aflatoxinas $B_{1}, B_{2}, G_{1}$ e $G_{2}$. Hoje conhecem-se, além destes mais seis metabólitos denominados $\mathbf{M}_{1}, \mathbf{M}_{2}, \mathbf{B}_{2 \mathrm{a}}, \mathbf{G}_{2 \mathrm{a}}, \mathbf{G M}_{1}$ e $\mathbf{B}_{3}$ (DUTTON e HEATHCOTE, 1969).

O substrato mais favorável é o amendoim, pelas peculiaridades de sua cultura e colheita, porém, a aflatoxina pode ser produziaja em praticamente todos os alimentos cuja umidade seja adequada para $o$ desenvolvimento do $A$. flavus.

A incidência da aflatoxina em nosso Estado tem sido grande como o demonstram os trabalhos de MENEZES et aiii (1966), TANGO et alii (1967) e FONSECA (1968).

Em virtude da importância do problema resolvemos aprofundar nosso estudo procurando conhecer qual o estágio da agro-indústria, dentro das condições prevalescentes em nosso Estado, em que havia maior produção de aflatoxina: se com o lavrador - desde a colheita até o momento da entrega do amendoim na fábrica — ou se nas mãos da indústria - durante o armazenamento da matéria prima e até o subproduto estocado que pode ser torta ou farelo. A região araraquarense foi escolhida pelo fato de que, em pesquisa por nós efetuada anteriormente (FONSECA, 1968) ter apresentado níveis gerais de aflatoxina mais elevados como também por ter sido mais elevada nesta região a incidência das aflatoxinas $\mathbf{G}$.

\section{MATERIAIS E MÉTODOS}

O material utilizado para execução deste trabalho constou de amostras de amendoim em casca e farelos de amendoim da safra das "águas" 
de 1967/68, coletadas na fábrica de óleo localizada no município de Matão. A fábrica e práticas agrícolas da região em que está situada tem as seguintes características predominantes. A fábrica tem capacidade de processamento de 30 toneladas de amendoim em casca por dia. O armazenamento da matéria prima ensacada é feito em armazens que a protegem eficientemente contra a possibilidade de reumidecimento.

A fábrica produz parte da matéria prima que industrializa e adota o seguinte procedimento: a planta é arrancada e "embandeirada", isto é, deixada com as sementes para cima no próprio local. Depois de alguns dias de seca, é procedida a batedura para separar as vagens das ramas, após o que é completada a seca em terreiro. Os lavradores em geral arrancam o amendoim com auxílio de sulcador ou bico-de-pato, deixam-no "embandeirado" na leira e tão logo seja possível batê-lo, fazem-no, ensacando logo em seguida.

As amostragens foram feitas nas seguintes épocas:

\begin{tabular}{|c|c|c|c|c|c|}
\hline Época I: & $12 / 2 / 1968$ & (amenc & loim & en & casca) \\
\hline Epoca II: & $4 / 3 / 1968$ & $"$ & & $"$ & " \\
\hline Época III: & $25 / 3 / 1968$ & $"$ & & $"$ & $"$ \\
\hline ca IV: & $2 / 4 / 1968$ & $"$ & & $"$ & $"$ \\
\hline a V: & $2 / 5 / 1968$ & (farelo & $\mathrm{de}$ & & ndoin \\
\hline oca VI: & $20 / 5 / 1968$ & & & & \\
\hline
\end{tabular}

Em cada Época foram coletadas 10 amostras numeradas, pela ordem de recolhimento, de 1 a 10, dando um total geral de 60 amostras.

Nas Épocas I e II as 10 amostras foram retiradas de 10 caminhões diferentes, à medida que iam chegando à fábrica. De cada um deles foi retirada uma amostra média, representando, no mínimo 10 sacos. Estas duas Épocas compreendem o Estágio 1.

Nas Épocas III e IV as amostras foram tomadas da matéria prima estocada nos armazens tendo-se procurado retirar parcelas do maior número de sacos possível. Estas Épocas compreendem o Estágio 2.

Nas Épocas V e VI foi usado o mesmo critério anterior, porém foram colhidas amostras de farelo de amendoim. Estas duas Épocas compreendem o Estágio 3.

Cada amostra de amendoim em casca foi constituída de aproximadamente $5 \mathrm{~kg}$ e as de farelo, entre 2 e $3 \mathrm{~kg}$.

As amostras foram recolhidas em sacos plásticos e identificadas.

As amostras de amendoim em casca foram descascadas e as amêndoas trituradas em máquina de moer carne, bem homogeneizadas e peneiradas em peneiras de crivo de 1680 micra (10 "mesh") e a seguir desengorduradas em extrator de Soxhlet. Os farelos foram também triturados em moinho de discos e peneirados. 
A toxina foi extraída de acordo com o método de LEE (1965), e dosadas pelo método de COOMES e FEUELL (1965). As amostras foram analisadas em triplicata e os resultados expressam a média obtida nas três determinações.

Para enquadramento das amostras quanto à sua toxidez foi utilizada a tabela do TROPICAL PRODUCTS INSTITUTE (1962) e que está baseada nos efeitos em testes biológicos levados a efeito com marrequinhos de um dia (QUADRO I).

QUADRO I - Relação entre concentração de aflatoxina $B_{1}$ e toxidez.

\begin{tabular}{l|l}
\hline Nivel de aflatoxina $B_{1}$ & Categoria de toxidez \\
\hline Abaixo de $0,05 \mathrm{ppm}$ & Baixa ou negativa \\
Entre 0,05 e $0,25 \mathrm{ppm}$ & Média \\
Entre 0,25 e $1,00 \mathrm{ppm}$ & Alta \\
Acima de $1,00 \mathrm{ppm}$ & Muito alta \\
\hline
\end{tabular}

Para a análise da variância dos resultados foram tomados os valores encontrados para a aflatoxina $B_{1}$ somados à metade dos valores de $G_{1}$ para cada amostra, visto que a aflatoxina $\mathrm{G}_{1}$ tem cerca de $50 \%$ da toxidez da $\mathrm{B}_{1}$ (CARNAGHAN et alii, 1963).

Estas variáveis (y) foram transformadas em $\log _{10}(y+1)$ de acordo com SNEDECOR (1956) e STEEL e TORRIE (1960) pois os valores encontrados eram de magnitudes muito diferentes e incluiam também os valores iguais a zero.

Foi feito também um estudo da evolução do teor de aflatoxina nas amostras em função do tempo, por meio de regressão. A soma dos Quadrados (SQ) para tempo foi desdobrada num componente linear, num componente de $2 .^{\circ}$ grau e em desvios de regressão. Paralelamente foram estimadas as constantes de uma equação de regressão do tipo:

$\log _{10}(\mathrm{y}+1)=\mathrm{a}+\mathrm{bx}+\mathrm{cx}^{2}$ (SNEDECOR, 1956).

onde $\mathrm{x}=\mathrm{n} .^{\circ}$ de dias a partir do início das coletas.

\section{RESULTADO E DISCUSSÃO}

Os resultados obtidos nas análises das aflatoxinas $B_{1}$ e $G_{1}$ encontram-se nos QUADROS II e III e os das análises estatísticas nos QUADROS IV, V e VI. 
QUADRO II - Teor das aflatoxinas $B_{1}$ e $G_{1}$ nas amostras de amendoim e farelo de amendoim nas diversas Epocas (expresso em ppm)

\begin{tabular}{|c|c|c|c|c|c|c|c|c|c|c|c|c|}
\hline \multirow{2}{*}{ Ainostra } & \multicolumn{2}{|c|}{ Epoca I } & \multicolumn{2}{|c|}{ Epoca II } & \multicolumn{2}{|c|}{ Epoca III } & \multicolumn{2}{|c|}{ Epoca IV } & \multicolumn{2}{|c|}{ Epoca V } & \multicolumn{2}{|c|}{ Época VI } \\
\hline & $\mathrm{B}_{1}$ & $G_{1}$ & $\mathrm{~B}_{1}$ & $\mathrm{G}_{1}$ & $\mathrm{~B}_{1}$ & $\mathrm{G}_{1}$ & $\mathrm{~B}_{1}$ & $G_{1}$ & $\mathrm{~B}_{1}$ & $\mathrm{G}_{1}$ & $\mathrm{~B}_{1}$ & $G_{1}$ \\
\hline 1 & 0,0 & 0,0 & 0,30 & 0,03 & 0,02 & 0,01 & 0,30 & 0,12 & 0,30 & 0,12 & 1,75 & 0,28 \\
\hline 2 & 0,0 & 0,0 & 0,08 & 0,03 & 0,08 & 0,03 & 0,75 & 0,66 & 0,02 & 0,01 & 0,75 & 0,28 \\
\hline 3 & 0,0 & 0,0 & 0,08 & 0,01 & 0,02 & 0,01 & 0,02 & 0,01 & 0,02 & 0,01 & 0,75 & 0,28 \\
\hline 4 & 0,0 & 0.0 & 0,08 & 0,01 & 0,0 & 0,0 & 0,30 & 0,0 & 0,02 & 0,01 & 0,75 & 0,28 \\
\hline 5 & 0,0 & 0.0 & 0,30 & 0,03 & 0.30 & 0,12 & 0.30 & 0,03 & 0,75 & 0,28 & 1,75 & 0,66 \\
\hline 6 & 0,75 & 0,0 & 0,08 & 0,01 & 0,02 & 0,01 & 0,30 & 0,03 & 0,75 & 0,28 & 0,75 & 0,28 \\
\hline 7 & 0,0 & 0,0 & 0,08 & 0,03 & 0,30 & 0,12 & 0,30 & 0,28 & 0,75 & 0,28 & 0,75 & 0,28 \\
\hline 8 & 0,0 & 0,0 & 0,02 & 0,01 & 3,75 & 1,41 & 0,75 & 0,28 & 0,75 & 0,28 & 0,75 & 0,28 \\
\hline 9 & 0,0 & 0,0 & 0,02 & 0,01 & 0,30 & 0,12 & 0,30 & 0,28 & 0,75 & 0,28 & 0,75 & 0,28 \\
\hline 10 & 0,0 & 0,0 & 0,08 & 0,03 & 0,30 & 0,12 & 0,30 & 0,12 & 0,75 & 0,28 & 0,75 & 0,28 \\
\hline
\end{tabular}

QUADRO III - Médias parciais e geral da aflatoxina $\left(B_{1}+1 / 2 G_{1}\right)$ por ÉPOCAS e por ESTÁGIOS, expressos em ppm.

\begin{tabular}{|c|c|c|c|c|c|c|}
\hline \multicolumn{7}{|c|}{ EPOCA } \\
\hline I & II & III & IV & $\mathrm{V}$ & VI & Geral \\
\hline 0,06 & 0,14 & 0,41 & 0,49 & 0,59 & 1,22 & 0,44 \\
\hline \multicolumn{2}{|c|}{$\begin{array}{c}\text { ESTÁGIO } 1 \\
0,08\end{array}$} & \multicolumn{2}{|c|}{$\begin{array}{c}\text { ESTÁGIO } 2 \\
0,45\end{array}$} & \multicolumn{2}{|c|}{$\begin{array}{c}\text { ESTÁGIO } 3 \\
0,88\end{array}$} & \\
\hline
\end{tabular}

QUADRO IV - Distribuição das amostras por níveis de aflatoxina $\left(B_{1}+1 / 2 G_{1}\right)$ e respectivas categorias de toxidez, em números absolutos (n) e percentagens.

\begin{tabular}{c|r|r|l}
\hline Niveis (ppm) & n & $\%$ & Categoria de Toxidez \\
\hline $00-0,05$ & 19 & 31,66 & Baixa ou Negativa \\
$0,05-0,25$ & 7 & 11,66 & Média \\
$0,25-1,00$ & 15 & 25,00 & Alta \\
$1,00-2,50$ & 18 & 30,00 & \\
$2,50-5,00$ & 0 & 0,00 & Muito Alta \\
$5,00-10,00$ & 1 & 1,66 & \\
Acima de 10,00 & 0 & 0,00 & - \\
\hline TOTAL & 60 & 100,00 & - \\
\hline
\end{tabular}


QUADRO V - Valores de $\mathrm{F}$ obtidos na análise da variância

\begin{tabular}{l|c|c}
\hline \multicolumn{1}{r|}{ Tratamento } & Graus de Liberdade & $\mathrm{F}$ \\
\hline \hline Epocas & $(5)$ & $8,03 * *$ \\
E. Estágios & 2 & $16,85 * *$ \\
D. Estágio 1 & 1 & 0,23 \\
D. Estágio 2 & 1 & 0,17 \\
D. Estágio 3 & 1 & $6,17 *$ \\
\hline
\end{tabular}

* Significância ao nível de $1 \%$ de probabilidade

** Significância ao nível de $5 \%$ de probabilidade

QUADRO VI - Valores de F referentes à aplicação de regressão no estudo do efeito de Epocas.

\begin{tabular}{l|c|c}
\hline \multicolumn{1}{r}{ Tratamento } & Graus de Liberdade & $\mathrm{F}$ \\
\hline Epocas & $(5)$ & $8,01 * *$ \\
Linear & 1 & $37,21 * *$ \\
Quadrático & 1 & 0,54 \\
Desvio Regressão & 3 & 0,77 \\
Resíduo & 54 & - \\
\hline
\end{tabular}

** Significância ao nível de $1 \%$ de probabilidade

Pode-se observar pelos resultados que, embora a maioria das amostras $(85 \%)$ apresentasse aflatoxina, o nível de incidência não foi muito elevado tendo sido praticamente nulo na Época I. Apesar disso, 31,6\% das amostras continham mais de $1,00 \mathrm{ppm}$, e portanto, na categoria de toxidez muito alta (QUADRO IV), especialmente na Época VI. Os níveis cresceram da Época I até a Época VI sem contudo atingir níveis excessivamente elevados pois estavam pouco acima de $1,00 \mathrm{ppm}$. As médias do teor de aflatoxina, em termos de toxidez total, cresceram de 0,06 a 1,22 ppm, com uma média geral de 0,44 ppm (QUADRO III).

A análise estatística revela que houve diferença significativa entre as Épocas e entre os Estágios, ambos ao nível de $5 \%$ de probabilidade apenas dentro do Estágio 3.

Os níveis mais baixos encontrados nas Épocas I e II são provavelmente devidos às boas práticas de secagem empregadas pela fábrica desta região visto que na coleta destas Épocas, predominaram amostras de amendoim produzido pela mesma ao passo que nas outras Épocas, as amostras analisadas eram na sua quase totalidade provenientes de matéria prima adquirida de lavradores da região. 


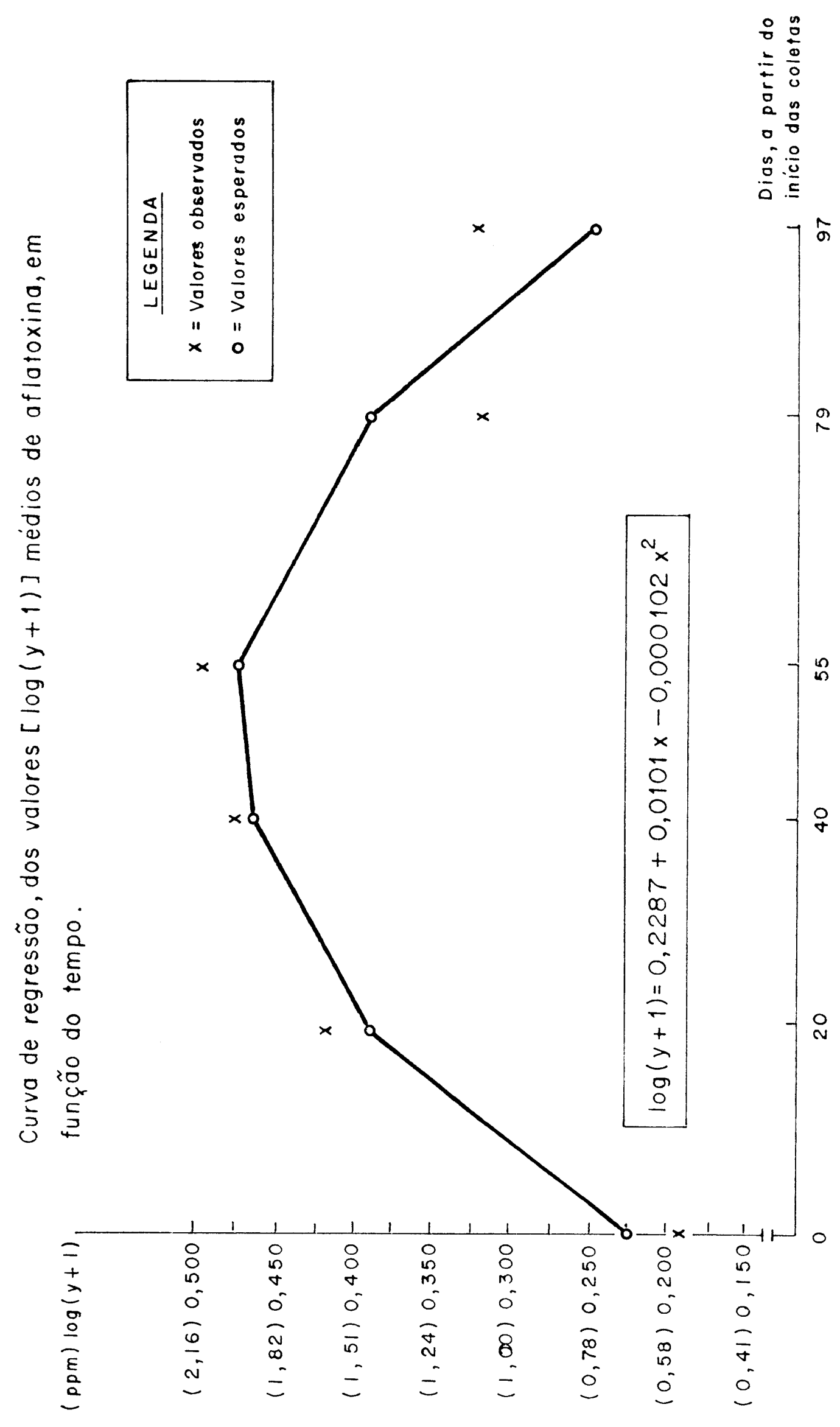


O estudo da variação da aflatoxina em função do tempo, empregando regressão, revelou que houve diferença entre as Épocas, significativa ao nível de $1 \%$ de probabilidade. Esta variação foi crescente e linear da Época I para a Epoca VI.

O valor de F, para o efeito linear, foi altamente significativo, ao nível de $0,1 \%$ de probabilidade, ao passo que para o quadrático não foi significativo (QUADRO VI). Isto indica que a evolução da ocorrência de aflatoxina nas amostras, em função do tempo, enquadrou-se numa equação do primeiro grau, ou seja, da reta (FIG. 1).

\section{CONCLUSÕES}

Dos resultados obtidos no presente trabalho pôde-se chegar às seguintes conclusões:

1) A maioria das amostras, representando $85 \%$ do total, continha aflatoxina.

2) O nível de aflatoxina nas amostras elevou-se continuadamente da Época I para a Época VI: médias de 0,6 até 1,22 ppm com média geral de 0,44 ppm.

3) As boas práticas de secagem da matéria prima antes do seu ensacamento e um bom armazenamento concorrem decisivamente para manter um nível muito baixo ou mesmo nuk de aflatoxina.

4) O lavrador entrega o amendoim à fábrica, já com aflatoxina.

SUMMARY

STUDY OF AFLATOXIN IN PEANUTS, FROM HARVEST TO

INDUSTRIALIZATION IN THE REGION OF MATÃO, S.P.

In the present work the occurrence of aflatoxin in peanut (Arachis hypoguea L.), in the region of Matão, S.P. was investigated, in three Stages, from harvest to industrialization: a) by the time the grower sells it to oil mill: Epochs $I$ and II; b) during its storage prior to milling: Epochs III and IV and c) after oil extraction (peanut flour): Epochs V and VI. In each stage two collections, of 10 samples each, were made in a total of 40 samples of peanuts and 20 samples of peaunt flour.

From the results the following conclusions could be drawn; a) the majority of the samples, representing $85 \%$, was toxic; b) the toxicity level of the samples, in terms of aflatoxin $\mathrm{B}_{1}$, grew from Epoch $\mathrm{I}$ to Epoch VI, presenting the average values of $0,06 \mathrm{ppm}$ in the first and $1,22 \mathrm{ppm}$ in the last one. The total mean value reached $0,44 \mathrm{ppm} ; \mathrm{c}$ ) good 
drying practices and proper storage can definitely help to maintain peanuts in a low level of aflatoxin and even prevent the occurrence of the toxin; d) the aflatoxin is already present in peanuts when growers take it to the oil mill.

\section{AGRADECIMENTOS}

Agradecemos à FAPESP pelo auxílio financeiro concedido e ao Prof. Roland Vencovsky pela orientação recebida na execução das análises estatísticas.

\section{LITERATURA CITADA}

ASPLIN, F.D. e R.B.A. CARNAGHAN; 1961. The toxicity of certain groundnut meals for poultry with special reference to their effect on ducklings and chickens. Vet. Rec. 73 (46) : 1215-19.

BLOUNT, W.P., 1960. Disease of turkey poults. Vet. Rec., 72(38) : 786.

BLOUNT, W.P., 1961. Turkey "X” disease. Turkeys, 9 (2) :52-55-8, 61, 67.

CARNAGHAN, R.B.A., R.D. HARTLEY e J. O'KELLY, 1963. Toxicity and fluorescence properties of the aflatoxins. Nature, 200:1101.

COOMES, T.J. e A.J. FEUELL, 1965. Recommended procedures for the detection and estimation of aflatoxin $B_{1}$ in groundnuts and groundnut materials. Tropical Products Institute, Report N. G13, Ministry of Overseas Development, Londres.

DUTTON, M.F. e J.G. HEATHCOTE, 1969. Some interesting relationship between the new aflatoxins and their associated metabolites. J. South African Chem. Inst. XXII: 5107-5118.

FONSECA, H., 1968. Contribuição ao estudo da ocorrência da aflatoxina em tortas, farelos e farinhas de amendoim (Arachis hypogaea L.) do Estado de S. Paulo. ESALQ/USP. Piracicaba, 65 p. Tese de Doutoramento.

HARTLEY, R.D., B.F. NESBITT e J. O'KELLY, 1963. Toxic metabolites of Aspergillus flavus. Bacteriol. Rev., 30(4):795-805.

LEE, W.V., 1965. Quantitative determination of aflatoxin in groundnut products. Analyst (Lond.), 90 (1070) : 305-7, 1965.

LOOSMORE, R.M. e J.D.J. HARDING, 1961. A toxic factor in Brazilian groundnut liver damage in pigs. Vet. Rec., 73(49):1362-4.

LOOSMORE, R.M. e L.M. MARKSON, 1961. Poisoning of cattle by Brazilian groundnut meal. Vet. Rec. $73(33): 64-5$.

MENEZES, T.J.B., J.S. TANGO, F.A.S. COELHO e C.G. TEIXEIRA, 1966. Ocorrência de Aspergillus flavus e da aflatoxina em sementes e farelo de amendoim. XVIII Reunião Anual da S.B.P.C., Blumenau, S.C.

SCHOENTAL, R., 1967. Aflatoxins. In: Annual Review of Pharmacology, 7 : 343-356.

SMITH, K.M., 1960. "Disease" of turkey poults. Vet. Rec., 72(32): 652. 
SNEDECOR, G.W., 1956. Statistical Methods. 5." ed., The Iowa State College Press. Ames, Ia. Cap. 5, 7 e 11.

STEEL, R.G.D. e J.H. TORRIE, 1960. Principles and Procedures of Statistics. Mc Graw-Hill, N. York, 481 p.

STEVENS, A.J.; C.N. SAUNDERS; J.B. SPENCE e A.G. NEWHAM, 1960. Investigations into "diseases" of turkey poults. Vet. Rec. 72(31):627-28.

SWARBRICK, O., 1960. Disease of turkey poults. Vet. Rec., 72 (33): 671.

TANGO, J.S., T.J.B. MENEZES e C.G. TEIXEIRA, 1967. Levantamento da ocorrência da aflatoxina em sementes de amendoim nas safras das águas e da seca de 1965. XIX Reunião Anual da S.B.P.C., Rio de Janeiro, R.J.

TROPICAL PRODUCTS INSTITUTE, 1962. Aflatoxin in groundnuts and groundnut products. Interpretation of physico-chemical and biological test results. T.P.I., Ministry of Overseas Development, Londres, 1 p 